\title{
Measurements of spatial resolution of ATLAS Pixel detectors
}

\author{
Tommaso Lari ${ }^{1}$ \\ Dipartimento di Fisica, Università di Milano and INFN, Sezione di Milano, via \\ Celoria 16, I-20133 Milano, Italy
}

\begin{abstract}
Standard as well as irradiated silicon pixel detectors developed for the ATLAS experiment were tested in a beam. Digital and analog resolutions were determined comparing the positions measured by a microstrip telescope and by the pixel detector. Digital resolutions of $10 \mu \mathrm{m}$ and analog resolutions of $6 \mu \mathrm{m}$ (before irradiation) and $10 \mu \mathrm{m}$ (after irradiation) are obtained without subtracting the error on the position determined by the telescope.
\end{abstract}

Key words: Spatial resolution. Silicon pixel detectors. Radiation hardness.

\section{Introduction}

The ATLAS Pixel sensors $[1,2]$ consist of $n^{+}$implants on a high resistivity $n$-bulk substrate which turns $p$-type after irradiation. The pixel dimensions are $50 \times 400 \mu \mathrm{m}^{2}$. Results on three different sensor designs are presented here: one with $p$-stop isolation between pixels [3], one with p-spray isolation [4] and one with $p$-spray isolation and the pixels surrounded by a floating $n^{+}$implantation. They will be referred as $p$-stop, new $p$-spray and old p-spray designs. The second is the nearest to final design for ATLAS.

1 On Behalf of the ATLAS collaboration [1]

Preprint submitted to Elsevier Preprint
The pulse height is obtained by the Time Over Threshold technique [1]. Typical thresholds were around 3000 electrons with a dispersion of 170 electrons rms. The noise was typically 150 electrons rms.

Several single chip assemblies were characterised extensively in test beam experiments [5]. Only resolution measurements are discussed here. A telescope consisting of 4 planes of silicon microstrips was used to reconstruct the tracks of the incident beam particles. The setup allowed the selection of the angle $\alpha$ between the normal to the pixel sensor plane and the beam direction.

Some sensors were irradiated to fluences comparable to those expected 
for LHC. Resolution measurements are presented for a $p$-spray device (old design) irradiated to $10^{15} \mathrm{n}_{\mathrm{eq}} / \mathrm{cm}^{2}$. Not irradiated sensors were operated at $150 \mathrm{~V}$ bias voltage achieving full depletion $(280 \mu \mathrm{m})$. The irradiated sensor was operated at $600 \mathrm{~V}$ corresponding to a depletion depth of 190 $\mu \mathrm{m}$ [5]. The average charge collected at the pixel centre for a perpendicular track was thus reduced from 28000 to 15000 electrons.

\section{Results}

In what follows, $x$ is the direction along the short $(50 \mu \mathrm{m})$ side of the pixel, $y$ the $400 \mu \mathrm{m}$ direction. Beam direction, the normal to the detector and the $x$ axis lie in the same plane.

The hit position can be reconstructed using only the position of fired pixels (digital algorithm). For cluster sizes greater than one it is possible to use also the analog algorithm which takes into account the correlation between the track position and the variable $\eta=Q_{l} /\left(Q_{l}+Q_{f}\right)$ where $Q_{f}$ and $Q_{l}$ are the charges collected by the first and last pixels in the cluster. The charge collected by other (central) pixels is not correlated to track position and so it is not considered. More details and a comparison with alternative algorithms can be found in [6] . The degradation of resolution due to Landau fluctuations is decreased by setting to a maximum value $Q_{\text {cut }}$ charges exceeding that limit.

Fig. 1 shows the $x$ residuals between position measured by the pixel detec-
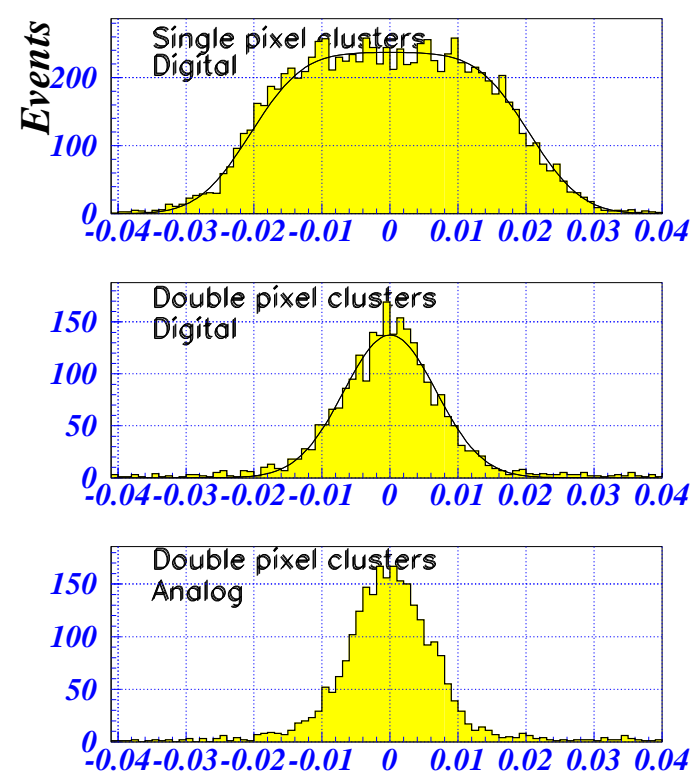

Position(mm)

Fig. 1. Residuals between position measured by the telescope and by pixel detector for the old $p$-spray device at $0^{0}$.

tor and by the telescope for the old $p$ spray device at $0^{0}$. At this angle $98 \%$ of the clusters have one or two pixels. The few events with higher multiplicity are due to delta-ray emission. At any given angle, analogously, there are always two dominant multiplicities. Residuals are plotted separately for each multiplicity.

Digital residual distributions are fitted with a square convoluted with a gaussian. The gaussian width is taken to be the telescope extrapolation error. Values of telescope resolution between 5.4 and $6.4 \mu \mathrm{m}$ are found for $\alpha \leq 10^{0}$. The square length is the range of track positions corresponding to that multiplicity. For old $p$ spray devices at $0^{0}$ this range is a $41 \mu \mathrm{m}$ interval centred on the centre of the pixel for single pixel clusters, a $9 \mu \mathrm{m}$ interval centred on the 
border between the pixels for double pixel clusters. The best digital resolution (Fig. 2) is obtained at angles for which the two dominant multiplicities are equally populated $\left(5^{0}\right.$ for not irradiated and $15^{0}$ for irradiated devices) while the worst resolution occurs when one of them is much more populated (as in Fig. 1).

At $5^{0}$ for not irradiated and $15^{0}$ for irradiated devices single and double pixel cluster occur with the same frequency and resolution is good. At $10^{0}$ for $p$-stop devices mainly double pixel clusters occur and resolution is worse. wermes@opala7.physik.uni-bonn.de

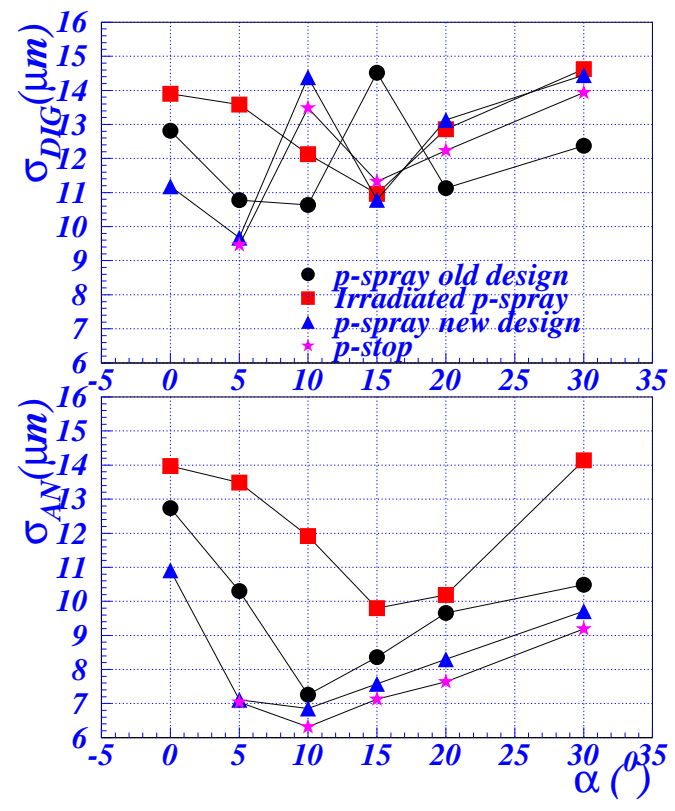

Fig. 2. Digital (above) and analog (below) residuals as a function of the angle of incidence of the beam, before subtraction of telescope resolution.

The best analog resolution (Fig. 2) is found at the angle at which mainly double pixel clusters occur. A resolution of $6.3 \mu \mathrm{m}$ is obtained at $10^{0}$ for the $p$-stop device which has the best charge collection, while a resolution of $9.8 \mu \mathrm{m}$ at $15^{0}$ is obtained for the irradiated sensor.

After deconvolution of the telescope error the digital resolution is always in the $(25 \div 50) / \sqrt{12} \mu \mathrm{m}$ interval, analog resolutions of $8 \div 12 \mu \mathrm{m}$ for the irradiated sensor and lower than $\sim 4 \mu \mathrm{m}$ for not-irradiated $p$-stop and new $p$-spray sensors at $10^{0}$ are found.

In the $y$ direction the residuals present a flat distribution between $-200 \mu \mathrm{m}$ to $+200 \mu \mathrm{m}$ with a rms of $115 \mu \mathrm{m}$. A bricked structure (with pixels in adjacent rows displaced by $200 \mu \mathrm{m}$ ) was also tested with the aim of improving the y resolution by a factor of two for clusters with charge sharing in the $x$ direction. Fig. 3 shows the $y$ resolution as a function of the angle. For $\alpha \geq 10^{0}$ all clusters have at least two pixels and the resolution reaches $65 \mu \mathrm{m}$.

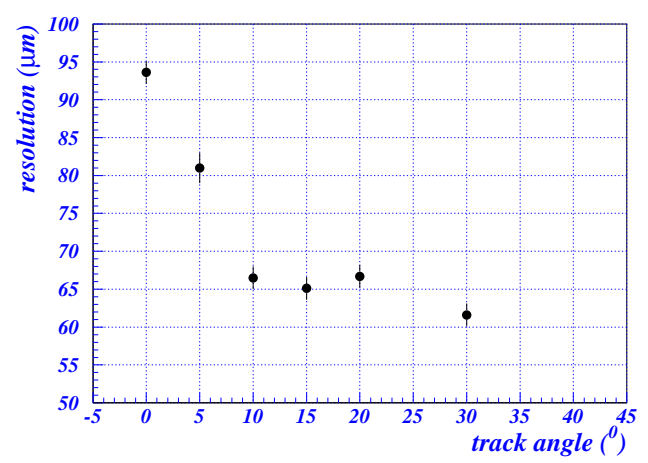

Fig. 3. Resolution in $y$ direction as a function of angle of incidence of the beam for the bricked design. 


\section{Conclusions}

Resolution measurements have been done on standard as well as irradiated prototypes of ATLAS Pixel sensors. In the short direction of the cell analog resolutions in the range 6-14 $\mu \mathrm{m}$ have been measured before subtraction of telescope resolution, estimated to be about $6 \mu \mathrm{m}$. A bricked design has been shown to improve the resolution in the long direction of the cell.

\section{References}

[1] The ATLAS Pixel collaboration, ATLAS Pixel Detector Technical Design Report, CERN/LHCC/98-13 (CERN 1998).

[2] M.S. Alam et al., The ATLAS silicon pixel sensors, CERN-EP/99152, submitted to Nucl. Instr. and Meth. A

[3] G. Batignani et al., Nucl. Instr. and Meth. A 277 (1989) 147.

[4] R. H. Richter et al., Nucl. Instr. and Meth. A 377 (1996) 412.

[5] F. Ragusa, Nucl. Instr. and Meth. A447 (2000) 184.

[6] C. Troncon, IEEE Trans. on Nucl. Sci. 47 (2000) 737.

R. Turchetta, Nucl. Instr. and Meth. A 335 (1993) 44. 